\title{
The Positioning Algorithm Research for Forest Fire Prediction
}

\author{
Dan Liu ${ }^{1}$ and Yanrong Zhang ${ }^{2}$ \\ ${ }^{1}$ Northeast Forestry University \\ ${ }^{2}$ Harbin University of Commerce \\ 531053387@qq.com, zhangyanrong_5@163.com
}

\begin{abstract}
In order to precisely predict the location of forest fire, a new algorithm based on improvement of gravity center scan method is introduced in this paper. The new algorithm suppressed error rate of InToOut and OutToIn. By using the neighbor nodes around the unknown nodes, it solved the problem of less signal flag nodes around unknown nodes, furthermore, it improved the coverage of nodes and suppressed error rate the location of node. The simulation result shows that, along with the increment of the density of signal flag nodes, the error rate of location of unknown nodes is gradually decreasing. In networks with limited signal flag nodes, the new algorithm based on improved gravity scan suppressed the error rate by $40 \%$, compared with previous gravity scanning algorithm, which dramatically improved the precision of location.
\end{abstract}

Keywords: wireless sensor network, Grest fire prediction, positioning algorithm, gravity scanning algorithm

Forest fire prediction focuses on prediction accuracy and fast prediction, you need to take advantage of wireless sensor positioning algorithm to reduce the positioning error rate. This article will study wireles sensor positioning algorithm by comparison and positioning algorithms to further enhance forest fire prediction the occurrence of positioning accuracy, thus providing a good algorithm to support forest fire forecast.

\section{Introduction}

Node positioning algorithms in wireless sensor networks have a lot. But it can really be applied to forest fire prediction is not much. Because external environment of the forest fire prediction is very special, and in order to increase the working life and spreading area of the sensor, requiring positioning algorithm to achieve low efficacy, low cost, high positioning accuracy. This paper describes these aspects in line with the focus of APIT (Approximate Point-in-trangulation Test) algorithms and positioning algorithm, and made on the basis of improyed gravity scanning algorithm.

\subsection{APIT Algorithm Defects}

APIT Location algorithm with low power consumption, low cost, high accuracy positioning node, and to achieve the principle is simple, easy features of the algorithm. So the positioning algorithm got a lot of applications. But in the process of the positioning algorithm, how to improve the algorithm of positioning error rate issues and determine whether the unknown node contains a beacon of nodes in the process, such as a triangle, mainly includes the following: 
(1) Theory of the PIT, if the unknown nodes near or just at the edge of the triangle, the positioning error will occur.

(2) Theory of the PIT, determined by the signal strength is within the triangle, will greatly increase the energy consumption of sensor nodes.

(3) The overlapping area calculating gravity, the accuracy is not high.

(4) If density of unknown nodes around the beacon node is big, positioning error rate is small. But if the density is small, the node can not be located, or positioning error rate is too high.

Therefore, based on the above algorithm problems, the researchers carried out various improvements to enhance the performance of the algorithm.

\subsection{Performance Comparison of Improved APIT Algorithm}

Literature [1]: The main improvement is unknown whether the node is included in a triangle and accuracy primarily by expanding the scope of the neighbor nodes, increase the number of neighboring nodes is unknown. Literature [2] introduced a coordinate influence coefficient estimates, improving the accuracy of node localization to solve the problem of sparse beacon nodes. Literature [3] from the security considerations nodes in the network, the nodes to achieve safe positioning ideas presented SAPIT algorithm. Literature [4] for sparse node localization inaccurate positioning algorithm proposed superinposed annular region ROCRSSI, the algorithm control signals are issued beacon nodes, node density connectivity and neighbors will not affect its error rate, but the algorithm is more complex.

Literature [5] used a triangle consisting of the vertical split in the four or six copies of a triangle using the strength of the detected signal narrowing targeted area, to improve the accuracy of node localization algorithm called PB-APIT.

Literature [6] using the grid all the way nodes randomly placed, so that the coverage of nodes similar uniform. Coverage areas is canied out to improve the node, so that the unknown nodes are distributed in a triangle composed of beacon nodes, the nodes to improve the positioning accuracy. Ireduces the probability of occurrence InToOut with OutToIn proposition, improve coverage beacon nodes. It proposed RAPIT, will receive the combined signal strength RSSI and APIT but greater consumption of other algorithms than the algorithm in terms of energy consumption. It proposed an improved IAPIT algorithm using the node and the communication radius ratio of the beacon to locate the node, to improve the positioning of coverage.

In more of these algorthms where each algorithm has its own characteristics, but after comparing the simulation algorithm, the center of gravity location algorithm for scanning PIT principle of two ropositions given a certain amount of interpretation, and in the calculation of the position of the unknown node the focus is on the use of stepwise refinement of methods to improve the positioning accuracy of the node, and low energy consumption in the process of calculation, the arithmetic is not complicated. So Proven comparison, the paper forest fire prediction localization algorithm for wireless sensor networks will be used to locate the center of gravity scanning algorithm, and the corresponding improvement for the algorithm deficiencies.

\subsection{APIT Test Theory Correction}

Before the gravity scanning algorithm was proposed, the first of two propositions PIT were corrected. 
Proposition 1 is amended as follows: If the unknown node $M$ does not exist away from or close to $A, B, C$ neighbor nodes located inside the neighbor nodes meet, then infer $M$ is inside, otherwise $M$ is located outside.

Specifically explained as follows: when the unknown node $M$ positioned within found to meet received from neighbor nodes $A, B, C$ beacon nodes to signal strength, and greater or less than $M$ is received from the $A, B, C$ to the signal strength, $M$ is located in an external front infer the need for the neighbor node $M$ receives the signal strength value for a judgment. If the signal strength is a good value is less than a relatively small value is set in advance based on experience, it means that the neighbor node is away from $M$, it may be externally, the test is not satisfied APIT theory. Therefore, no direct inference $M$ is located outside, and then the other neighbor nodes APIT tests.

Proposition 2 is amended as follows: When a node density is too low, only by increasing the number of nodes to improve the proposition.

According to amend these two propositions, during the focus positioning argathm, try to avoid OutToIn and InToOut these two errors, thereby improving the positioning accuracy.

\subsection{Achievement in the Gravity Scanning Algorithm}

Based on the above defects, the gravity scanning positioning algorithm introduced legal triangle, illegal triangle and scanning algorithm supported by data sets. The node to be positioned $M$ according to the broadcast signal strength of beacon nodes, determining the node associated with the $\mathrm{M}$ beacon node triangles composition. According to the theory of modified APIT test, $M$ neighbor nodes are in $\mathrm{N}$ triangles. If there are $\mathrm{L}$ triangles that include $M$ nodes, the $L$ triangles are legal triangle. It there are not $N-L$ triangles that include $M$ nodes, the $N-L$ triangles are illegal triangle. The $N$ triangle area is scanning algorithms to support data sets.

APIT has been improved by the triangle gravity scanning algorithm. After the improvements are given three definitions:

Definition 1: If node $M$ receives node $M^{\prime}$ signal strength is greater than a preset value, called nodes $M^{\prime}$ is a lêgal neighbor nodes; otherwise, $M^{\prime}$ is not legal.

Definition 2: APIT argorithm is the neighbor node to be positioned to receive broadcast messages for other unknown nodes.

Definition 3: APIT algorith to locate a neighboring node is the node can be other unknown node receives a broadcast message of its proximity and the beacon nodes.

According to the three definitions, APIT algorithm further refinement, to minimize the probability of OutTom and InToOut occur. The positioning of the unknown node M scanning algorithms to support data set.

The gravity scanning algorithm steps:

(1) According to the unknown node $M$ proceeds scanning algorithms to support data set, there are l legal areas of a triangle. The Formula 1-1 to calculate the coordinates of the center of gravity of each triangle, $\left(\mathrm{x}_{1}, \mathrm{y}_{1}\right),\left(\mathrm{x}_{2}, \mathrm{y}_{2}\right), \cdots,\left(\mathrm{x}_{L}, \mathrm{y}_{L}\right)$.

$$
\left\{\begin{array}{c}
x_{i}=\left(x_{i a}+x_{i b}+x_{i c}\right) / 3 \\
i=1 \cdots L \\
\begin{array}{c}
y_{i} \\
i=1 \cdots L
\end{array}=\left(y_{i a}+y_{i b}+y_{i c}\right) / 3
\end{array}\right.
$$

The coordinates of each point of the legal triangle is $\left(\mathrm{x}_{i a}, \mathrm{y}_{i a}\right),\left(\mathrm{x}_{i b}, \mathrm{y}_{i b}\right),\left(\mathrm{x}_{i c}, \mathrm{y}_{i c}\right)$, the value of $i$ is $1,2, \cdots, L$. 
(2) The coordinates of the center of gravity is calculated, $\left(\mathrm{x}_{1}, \mathrm{y}_{1}\right),\left(\mathrm{x}_{2}, \mathrm{y}_{2}\right), \cdots,\left(\mathrm{x}_{L}, \mathrm{y}_{L}\right), \mathrm{a}$ new bundle of triangular region. If, put the remaining one or two nodes directly under a center of gravity coordinates are included.

(3) Repeats Step (1) and (2) to calculate the center of gravity of each triangle, cycle calculates the center of gravity, until no longer able to form a triangle so far, which is calculated by one to two gravity.

(4) The remaining one coordinate is directly coordinate unknown nodes, two coordinates do a centroid algorithm to obtain the coordinates of the unknown nodes.

By implementing the above steps and algorithms can be concluded that the algorithm is computationally simple, you only need beacon nodes can coordinate and calculation step is not complicated. So the higher the efficiency, consuming less energy, these advantages to be good with the need to locate the system in this article, but the positioning method remains inadequate:

(1) When a node uneven distribution of less unknown node to be positioning beacon nodes surrounding the case, the localization algorithm will produce large errors rates. Because of unknown nodes positioned around the beacon nodes to meet the rêquirements of the triangle less, and no stepwise refinement, so the case of uneven distribution of beacon nodes, higher positioning system error rate, which is the disadvantage of APIT algorithm.

(2) According to the algorithm is given in the received signal strength of the beacon nodes to determine whether the unknown node beacon nodes around the triangle inside. But it did not give a specific determination method. The paper will be based on the specific practice application gives the judgment threshold.

Based on these two shortcomings, the focus of this paper, based on the scanning location algorithm is proposed to improve the ways in which the nodes are able to more accurately locate the nodes laid (n) any way to improve the positioning accuracy and reduce the positioning error rate.

\section{Improved theory of Gravity Scanning Localization Algorithm}

Forest fire prediction focuses on accuracy and fast responding. Gravity scanning location algorithm given a centan interpetation for two propositions of PIT principle and perform stepwise refinement methods to calculate the location of unknown node [1]. It improves the location accuracy of the hodes decreases energy consumption of calculation by using the simple arithmetic. After comparison and validation, Gravity scanning location algorithm will be used in forest fire prediction localization algorithm of wireless sensor networks in this paper, and the disadvantage will be improved.

There are two theories to improve two disadvantages of gravity scanning location algorithm. Those two methods will be demonstrated in detail and be implemented in the improved argorithm which enhances the value of the application.

Theory 1. When the count of the beacon nodes around an unknown node is less than 5 , the loeation of the node is calculated by the location of neighbor nodes based on beacon nodes.

Argument: the theory is mainly to solve the low density of beacon nodes around target node. The accuracy of location will not be well controlled when less beacon nodes surround target node. Positioning error will be induced in this situation. 


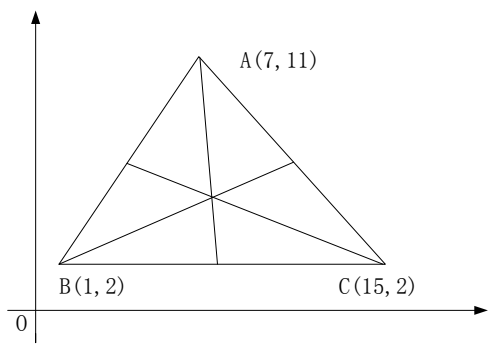

Figure 1. Three Beacon Nodes to Calculate the Barycentric Coordinates

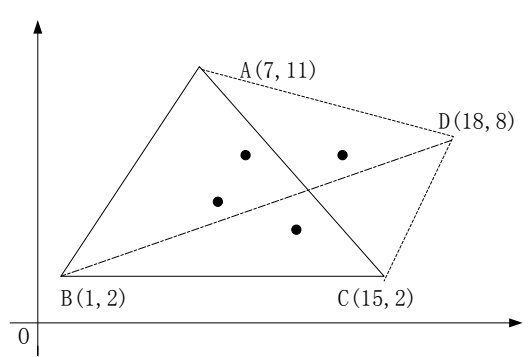

Figure 2. Four Beacon Nodes to Calculate the Barycentric Coordinates

\subsection{In Figure 1., there are 3 Beacon Nodes Around Unknown Node}

The coordinate of 3 beacon nodes are: $\mathrm{A}(7,11), \mathrm{B}(1,2), \mathrm{C}(15,2)$, gravity location is $(7.67,5)$ by using gravity location algorithm. However, error is increased significantly which is due to unknown node may be located at any position in the larger triangle area.

\subsection{In Figure 2., there are 4 Beacon Nodes Around Onknown Node}

There are $C_{4}^{3}$ triangles calculated by gravity seanning positioning algorithm when unknown node could receive signal from 4 beacon nodes. One new triangle will be created by three new nodes which are the gravity randonly selected from 4 triangles. The gravity of new triangle and the rest one node will be used to calculate the location of unknown node by centroid algorithm. See Figure 2.

Unknown node will be localized oy 4 beacon hodes. One more beacon node $\mathrm{D}(18,8)$ combines with other 3 beacon nodes. The gravity in 3 triangles is: $(7.67,5),(13.3,7)$, $(11.3,4),(8.67,7)$.The interested area is getting smaller and final estimated location is $(10.235,5.75)$. So the devian between loctimated location and actual location is closely.

\subsection{In Figure 3., there are 5 Beacon Nodes Around Unknown Node}

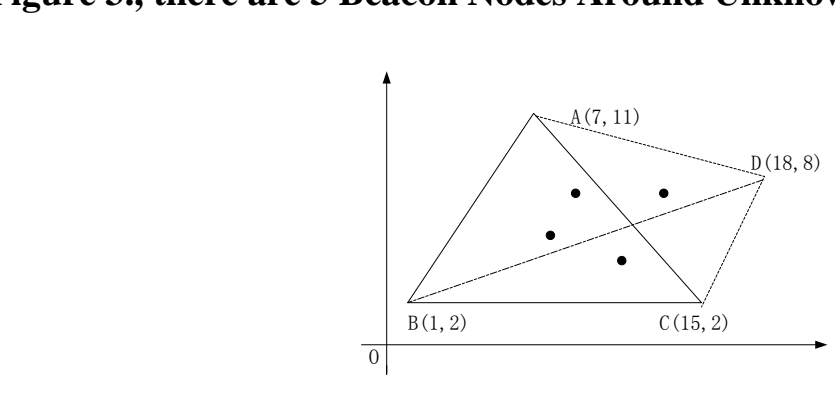

Figure 3. Five Beacon Nodes to Calculate the Barycentric Coordinates

There are $C_{5}^{3}$ triangles by 5 beacon nodes. But not all triangles include the unknown node. Few triangles are besides the unknown nodes. However, $\triangle B D E, \triangle A C D, \triangle A C E$ still include the unknown node. Positioning accuracy is increased due to interested area is decreased.

Based on above figure descriptions and data illustration, when beacon nodes around an unknown node is less than or equal to five, the error rate will be relatively high, because fewer number of triangles surrounded by beacon nodes, the area is larger by gravity formation which induces low positioning accuracy, when beacon nodes around an unknown node is greater than or equal to six, the density of beacon nodes is increasing, the area including 
unknown node is smaller. Positioning accuracy is high. When the beacon nodes is less than six and the count cannot be added since the density of beacon node is not averagely, neighbor nodes will be used for the calculation[2-3]. The location of neighbor will be calculated firstly since neighbor may be another unknown node. Thereby the location of target node is known.

Theory 1 solves the uneven distribution of beacon nodes. Less beacon nodes surrounds unknown node will induce low successful rate of location accuracy.

Theory 2: Summarizes the number of nodes besides triangle area by beacon nodes and accumulates signal strength. The average value as a threshold value, the unknown node is determined outside the triangle if the unknown signal strength is less than this threshold.

Argument: In the gravity positioning algorithm, each node must be determined if it is included in the triangle by beacon nodes, but it is difficult for internal calculations, boundary values or error happen usually. Therefore, each node will be determined whether it is an external point from triangle, then further argues that in order to address APIT positioning algorithm OutToIn misjudgment problem [4].

External node will be found by signal strength, and that kind of node will be statistical by count $N$. Signal strength will be calculated for each node based on the triangle created by beacon nodes. $M / n$ is threshold value; $\mathrm{M}$ stands for the accumulation of all signal strength. Internal judgment is performed on beacon nodes, signal strength will be compared with threshold value. Unknown node will be outside of triangle if signal strength is less than threshold. This solves the mistake in OutToIn. Above two theories will significantly improve the OutToIn and InToOut issues in gravity scanning positioning algorithm [5]. They reduce error rate and enhance the accuracy.

\section{The Basic Idea of improved Gravity Scamning Algorithm}

Improved gravity scanning algonth $\mathrm{m}$ is using theory 1 and theory 2 . The count and location of unknown nodes will be calculated before real positioning algorithm. Theory 1 solves the low density beacon nodes around unknown node. Theory 2 solves the accuracy of relative position between unknown node and triangle composed by beacon nodes. Two theories provide a good theoretical basis for the positioning accuracy [6].

The basic idea of improved granty scanning algorithm is as follows:

(1) Unknown node $M$ records the coordination of all beacon nodes around them and count the number based on the sigralbroadcast.

(2) The number of beacon nodes is by using improved gravity localization theory 1.If $n>5$, localization win be calculated directly. If $n \leq 5$, have to use location of unknown neighbor nodes to localize the position of unknown node. Unknown neighbor node will be new beacon node.

(3) $C_{n}^{3}$ Triangles will be created by beacon nodes around unknown node. $M$ will be judged by gravity argorithm to known if it locates in the triangle. In the progress, high accuracy will be reached by utilizing improved theory 2. Reasonable triangle will be counted as $m$.

(4) Gravity scanning localization algorithm will be performed on the beacon nodes in the reasonable triangles. Estimate the location of unknown node.

By describing the algorithm in the process of implementation, if the number of unknown nodes around the beacon nodes is greater than 5, then the algorithm complexity is consistent with gravity scanning algorithm, not a lot of energy consumption during operating; However, if the number of beacon nodes around the unknown node is less than or equal to 5, the algorithm will increase the complexity but the network does not have much impact on energy consumption since the major part of the algorithm is calculation. Communication of data will 
be 2 time higher than before but not an over loading for the network. Therefore, an improved localization is suitable and efficacy for forest fire prediction system on WSN.

\section{Realization of the improved Gravity Scanning Localization Algorithm}

The concrete realization steps of the algorithm are shown as follows:

(1) According to the broadcast signal regularly sent among different nodes, use $\mathrm{n}$ to record the amount of beacon nodes which is received by unknown node $M$, if $n>5$, go to step (3); otherwise, use $\mathrm{m}$ to record all the neighbor nodes except the beacon nodes of node $\mathrm{M}$, the nodes set can be presented as $\left\{\mathrm{D}_{1}, \mathrm{D}_{2} \cdots, \mathrm{D}_{m}\right\}$.

(2) Operate the neighbor nodes according to step (1).

(3) Set reasonable triangles composed by nodes which meet the gravity scanning localization algorithm and the improved algorithm, calculate the gravity of each triangle, execute step (2) to step (4) gravity scanning algorithm to estimate the location of anknown nodes.

The Pseudo code of the improved center-of-gravity scanning localization algorithm is shown as follows:

Receive location beacons $\left(\mathrm{x}_{i}, \mathrm{y}_{i}\right)$ from $\mathrm{n}$ anchor

If $(\mathrm{N}<=5)\{$

Receive location neighbor nodes $\left(\mathrm{x}_{i}, \mathrm{y}_{i}\right)$ from $\mathrm{m}$ anchors;

InsideSet $=\phi \quad$;

for(each triangle $\mathrm{Ti} \in$ triangles $)\{$

if (Point-In-Triangle-Test(Ti)==TRUE)

InsideSet=InsideSet $U\{\mathrm{Ti}\}$;

if(accuracy(InsideSet)>enough)break; \} .

Estimated Position=Center Of Gravity $(\cap$ Ti - InsideSet);

\section{The Simulation Realization of the improved Gravity Scanning Algorithm}

Use Matlab7.0 as the simulation tool, nodes amount of simulation area can be adjusted at any time according to the actual needs, nodes are randomly distributed in the area of $100 \mathrm{~m} * 100 \mathrm{~m}$, compare the simulation experiments of APIT algorithm, gravity scanning positioning algorithm and the improved gravity scanning positioning algorithm, and the results are shown as follows.

\subsection{Comparison of Positioning Error Rate}

The distribution density of beacon nodes is the main factor to influence the positioning error. Ouring the simulation process, randomly set 100, 200 and 300 nodes in a fixed area, respectively simulate APIT algorithm, gravity scanning positioning algorithm and the improved gravity scanning positioning algorithm in the area, distribution density of beacon nodes gradually expands from $1 \%$ to $70 \%$, locate each unknown node and calculate the positioning error, draw the simulation curve as Figure 4, Figure 5 and Figure 6.

(1) Positioning error simulation of 100 nodes distributed randomly.

Whether a node is included in the triangle composed by beacon nodes can appear the miscalculation of OutToIn and InToOut when using APIT algorithm to locate unknown nodes, which results in the node positioning error. In addition, under the situation of sparse density 
of beacon nodes around unknown nodes, the localization accuracy is relatively low. Therefore, positioning error rate will gradually become stable when density of beacon nodes is $25 \%$, otherwise, positioning error rate is high.

Gravity scanning positioning algorithm solves parts of the problem of OutToIn and InToOut miscalculation, however, it does not consider the localization accuracy problem caused by too little beacon nodes around unknown nodes and constituent triangles. Therefore, the positioning error rate of gravity scanning positioning algorithm is lower than APIT, however, error rate is still relatively high under $25 \%$ of beacon nodes.

The improved gravity scanning positioning algorithm uses the reverse way based on gravity scanning localization algorithm to solve the problem of OutToIn and InToOut miscalculation, and uses the iterating way to calculate the coordinates of neighbor nodes around the unknown nodes when the amount of beacon nodes is lower than 5, it expands the amount of nodes with coordinates, which can resolve the problem of high positioning error rate in the situation of sparse density of beacon nodes. It can be seen from Figure 4 that the algorithm still has low positioning error rate with sparse density of beacon nodes, the reduce of positioning error rate can be $50 \%$ in the situation that percent of beacon nodes is lower than $25 \%$.

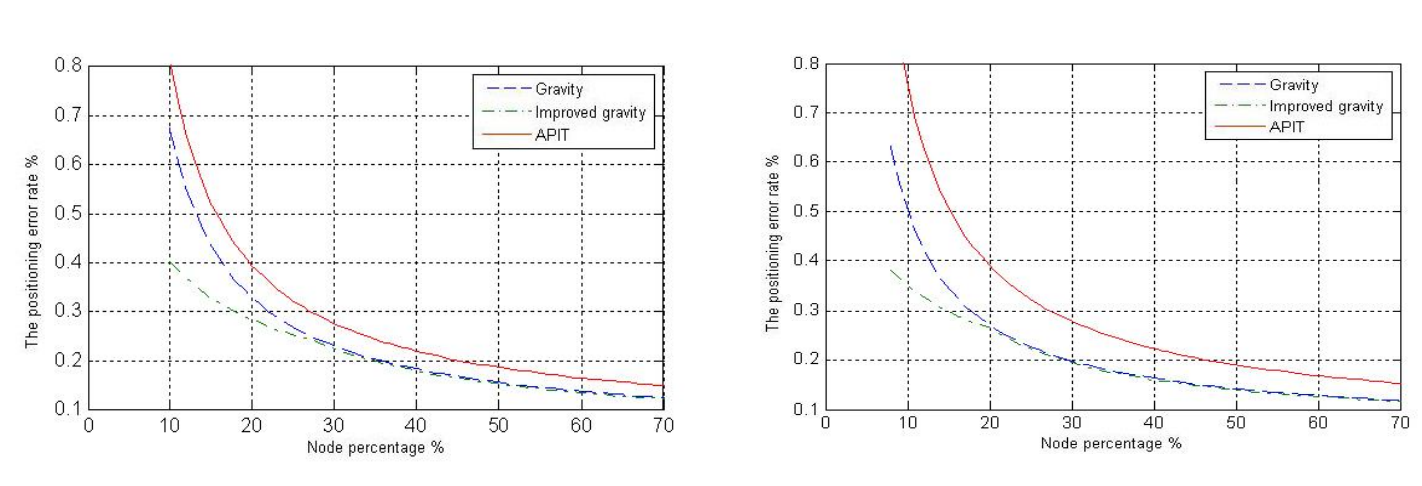

Figure 4. The Total Number of Nodes of 100 of the Positioning Error Rate

Figure 5. The Total Number of Nodes of 200 of the Positioning Error Rate

(2) Positioning error simulation of 200 nodes distributed random.

As the amoun increase of andomly distributed nodes, the distribute density of beacon nodes is gradually expanding, the positioning error rate becomes stable when the density of beacon node arrives at $20 \%$. The improved gravity scanning positioning algorithm decreases $20 \%$, but the other two algorithms are still high.

(3) Positionling error simulation of 300 nodes distributed randomly.

As the amount increase of randomly distributed nodes, the distribute density of beacon nodes I gradually expanding, the positioning error rate becomes stable when the density of beacon node arrives at $15 \%$. The improved gravity scanning positioning algorithm decreases $15 \%$ but the other two algorithms are still high.

The simulation results show that the positioning error rate is mainly determined by the density of beacon nodes, positioning error rate is low when the beacon nodes density is high, and positioning error rate is high when the beacon nodes density is low. Through setting 100 , 200 and 300 nodes in a fixed area, positioning error is gradually decreasing as the density of beacon nodes is gradually increasing of the three algorithms. However, the improved gravity scanning positioning algorithm improves the problem of OutToIn and InToOut miscalculate, and introduces the neighbor nodes to help when the beacon nodes is sparse around the 
unknown nodes, therefore, positioning error rate reduces about $50 \%$ of the other two algorithms under the sparse density of beacon nodes. The proposed algorithm is better than other algorithms in positioning accuracy and it has a good application value.

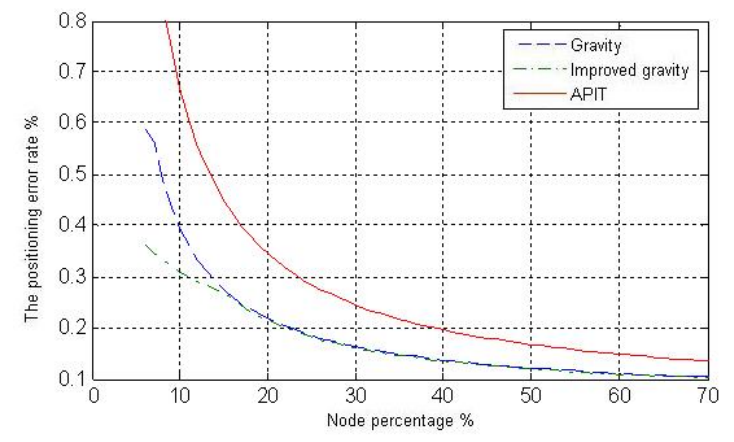

Figure 6. The Total Number of Nodes of 300 of the Positioning Error Rate

\subsection{Comparison of Positioning Error and Coverage Rate}

Environment of the simulation does not change and set 20110 nodes in a fixed area, percentage of beacon nodes is fixed $30 \%$, carry out simulation experiments of APIT algorithm, gravity scanning localization algofithm and the jimproved gravity scanning positioning algorithm, and the results are shown as Figure 7 and Figure 8.

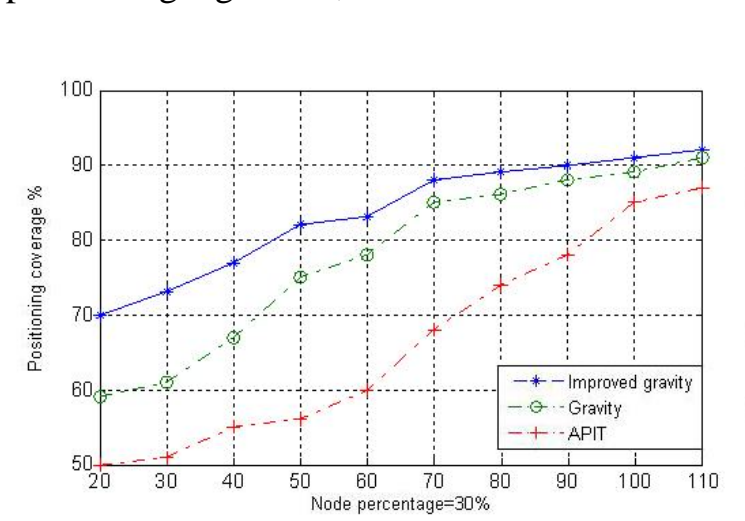

Figure 7. Positioning Coverage of $30 \%$ Beacon Node

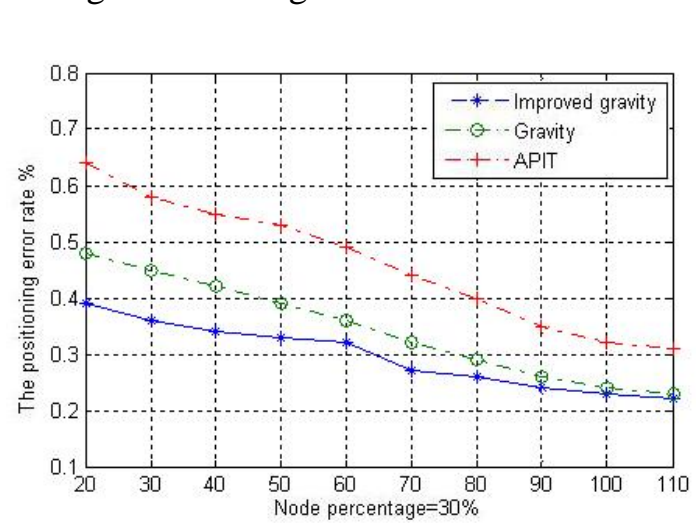

Figure 8. Positioning Error Rate of $30 \%$ Beacon Node

According to the features of the above three algorithms, node coverage rate of APIT is lowest, especially under the situation of litter amount of nodes and the coverage rate can not arrive at $50 \%$. As the amount of nodes increases, the coverage rate is improving. Node coverage rate of gravity scanning positioning algorithm increases by $20 \%$ comparing with APIT after solving parts of the problem of OutToIn and InToOut miscalculate. The improved gravity scanning positioning algorithm solves the problem of OutToIn and InToOut miscalculate and uses neighbor nodes around unknown nodes to increase node coverage rate, therefore, it has a relatively high coverage rate and increases by $20 \%$ than APIT algorithm. The results of node positioning rate are the same as above, positioning error rate decreases as the nodes amount increases.

Simulation results show that error rate of each algorithm is gradually reducing and coverage rate is improving as the unknown nodes is increasing, the improved gravity 
scanning positioning algorithm has the best performance among the three algorithms, problem of sparse density of nodes has been solved and positioning accuracy of unknown nodes has been improved, which has provided a good algorithm for network node localization.

\section{Conclusion}

In order to accurately predict the location of forest fires occurred in the center scan positioning algorithm based on the improvement, the improved algorithm reduces the false positive rate of InToOut and OutToIn of neighbor nodes around the unknown node to solve the unknown node around the beacon node sparse problems and improve the coverage of the node, to reduce the error rate of node localization. Simulation results show that the unknown node location error rate gradually decreased with the increase in the density of beacon nodes; compared with the original center scan localization algorithm, the localization error rate of the improved center scan localization algorithm fell by about $40 \%$ in a sparse network, greatly enhance the localization accuracy of the network, and greater flexibility for the positioning of randomly distributed wireless sensor nodes, and more advântage than other positioning algorithms.

\section{Acknowledgements}

This paper is supported by "the Fundamental Research Funds for the Central Universities"-----2013CBQ01.

\section{References}

[1] J. Zeng, W. Hong and X. Jin, "Improyement on APIT loc dization algorithms for wireless sensor networks", Proceedings of the 2009 International Conference on Networks Security,Wireless Communications and Trusted Computing, vol. 1, (2009) pp. 719-723.

[2] Z. Zhong, D. Y. Luo, S. Q. Liu and X. P Fan An adaptive localization approach for wireless sensor networks based on Gauss-Markov mobility 'model', Acta Automatica Sinica, vol. 36, no. 11, (2010), pp. 1557-1568.

[3] H. Y. Chen, Q. J Shi and R. Tan, “Mobileelement assisted cooperative localization for wirelesssensor networks with obstacles,, IEEE Transactions on Wireless Communications, vol. 9, no. 3, (2010), pp. 956963.

[4] X. H. Kuang, H H Shao and R. Feng, "A new dis-tributed localization scheme forwireless sensor networks", Acta Automatica Sinica, vo1 34, 10. 3, (2008), pp. 344-348.

[5] G. J. Wang, T. Wang and W. J Jia, "Adaptive location updates for mobile sinks in wireless sensor networks", Journal of Supercomputing, vol. 47, no. 2, (2009), pp. 127-145.

[6] R. B. Zhang, L. L.Zhang and Y. B. Feng, "Very low energy consumption wireless sensor localization for danger environments with single sensor mobile anchor node", Wireless Personal Communications, vol. 47, no. 4, (2008),pp. 497-521

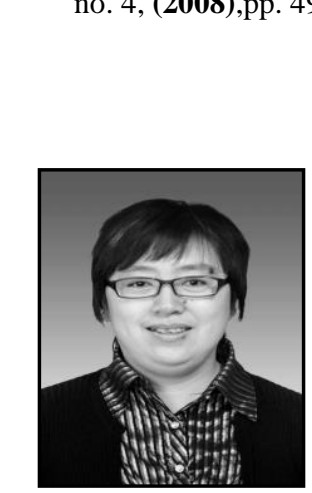

\section{Author}

Dan Liu, Doctor, Lecturer, teacher of School of the Information and Computer Engineering College, Northeast Forestry University. Her main research fields are Wireless Sensor Networks and Artificial Intelligence. 